\title{
Effects of Carbonic Anhydrase Inhibitor Acetazolamide (AZ) on Osteoclasts and Bone Structure
}

\author{
Chihiro Shinohara $^{1)}$, Kikuji Yamashita ${ }^{2)}$, Takashi Matsuo ${ }^{3)}$, Seiichiro Kitamura ${ }^{2)}$ and Fumiaki Kawano ${ }^{1)}$
}

\begin{abstract}
1) Department of Oral Care and Clinical Education, Tokushima University Hospital
2) Department of Oral and Maxillofacial Anatomy, Institute of Health Biosciences, The University of Tokushima Graduate School

${ }^{3)}$ Department of Conservative Dentistry, Institute of Health Biosciences, The University of Tokushima Graduate School
\end{abstract}

(Accepted for publication, July 24, 2007 )

\begin{abstract}
We examined the changes of osteoclasts and bone structure in vivo and in vitro under the condition of low activity of bone resorption induced by acetazolamide (AZ). AZ is a specific inhibitor of carbonic anhydrase II (CA II), which is the enzyme indispensable for substantial proton generation by osteoclasts. In vivo study, we investigated histologically and histomorphometrically the changes of trabecular bone and osteoclasts at the cartilage-bone junction of tibiae in rats injected subcutaneously with AZ for 21 days. The area of trabecular bone of AZ treated group increased compared with control group. Moreover, AZ treatment decreased about $20 \%$ the number of osteoclasts at the cartilage-bone junction. In vitro study, we examined the effects of AZ on osteoclasts using culture system of rat bone marrow. The number of osteoclasts on the bone slice was suppressed by $\mathrm{AZ}$ of a concentration from $10^{-5}$ to $10^{-4} \mathrm{M}$ in a doze-dependent manner. The number of osteoclasts cultured with $10^{-4} \mathrm{M}$ AZ for 24 hours and with or without parathyroid hormone (PTH) on the bone slice and on the glass showed a significant decrease compared with the control group. Some osteoclasts treated with AZ showed morphological changes of apoptosis-like cell death, including shrinking of cytoplasm and fragmentation of nuclei. These results show that high-doze of AZ decreases bone resorption not only by inhibiting CA II but also by decreasing the number of osteoclasts via induction of cell death. This suggests that CA II plays an important role not only in proton formation but also in osteoclast survival.
\end{abstract}

Key words : Acetazolamide, Bone, Carbonic anhydrase II, Osteoclast

\section{Introduction}

Osteoblasts and osteoclasts maintain bone homeostasis in a coordinated manner. Their differentiation and function are regulated by hormones and cytokines, and aberration of their cells causes metabolic bone diseases such as osteoporosis, osteopetrosis, paget's disease of bone and so on. Additionally, it is revealed M-CSF (macrophage colony stimulating factor) and RANK [receptor activator of nuclear factor (NF)-kB]/RANKL (RANK ligand) system are essential for cell-cell interaction between osteoblast-lineage cells and osteoclast-lineage cells ${ }^{1-3)}$. However, clarification of each cell function is still incomplete.

Osteoclasts perform their function of resorbing bone by adhering to bone via integrin receptors, dissolving bone matrix minerals using protons, and degrading organic materials with hydrolytic enzymes intra- and extracellularly ${ }^{4)}$. Protons are produced from water and carbon dioxide by the activity of carbonic anhydrase II (CA II) at the cell membrane ${ }^{5)}$ and are

Corresponding author: Chihiro Shinohara, Department of Oral Care and Clinical Education, Tokushima University Hospital 3-18-15 Kuramotocho, Tokushima 770-8504, Japan, Tel.: +81-(0)88-633-9181 Fax: +81(0)88-633-9182.E-mail address: chihiro@dent.tokushima-u.ac.jp actively transported into resorption pits through V-ATPase (proton pump) at the surface of ruffled border ${ }^{6}$. Proton production by CA II is indispensable for bone resorption and congenital defects in CA II function are known to cause human osteopetrosis ${ }^{7)}$. Though acetazolamide (AZ), a sulfonamide inhibit CA II specifically and suppress bone resorption ${ }^{8)}$, the influences in the survival of osteoclasts and osteoblasts are not clear. For that reason, the effects of AZ in the trabecular bone structure, the amount of osteoclasts and their morphology were examined at the cartilage-bone junction in tibiae of rats injected subcutaneously with AZ. We also analyzed the effects of $\mathrm{AZ}$ in the bone resorption by rat osteoclasts examining resorption pits, number of osteoclasts and their shape among bone marrow cell culture. In vitro study, we used parathyroid hormone (PTH) and bone slices to investigate the changes of osteoclasts in a similar environment to living body. Furthermore we used coverslips to observe the morphology of osteoclasts clearly.

\section{Materials and Methods}

\section{In vivo examination}

Five-week-old male Sprague Dawley rats (Nihon Charles 
Chihiro Shinohara et al.: Effects of acetazolamide on osteoclasts and bone

River, Kanagawa, Japan) were injected subcutaneously with AZ (Sigma Chemical Co., St. Louis, MO) or vehicle dimethylsulfoxide (DMSO, Wako Pure Chemical, Osaka, Japan) at a dose of 50 mg/ day per kgBW. The tibiae were removed on day 5, 7, 14 and 21, fixed in 10\% formalin, demineralized with 10\% EDTA, embedded in paraffin and cut at $5-\mu \mathrm{m}$ in longitudinal sections. Three serial sections were placed on each slide glass. Each five slide glasses from the central portion of the tibiae, separated by an interval of 4 slide glasses, were stained with hematoxylin and eosin (HE) and tartrate-resistant acid phosphatase (TRAP), a typical enzyme marker of osteoclasts. Changes in trabecular bone were examined by HE staining, and the ratio of bone per unit area $(290 \mu \mathrm{m} \times 255$ $\mu \mathrm{m})$ subjacent to the epiphyseal plate was measured using a videomicrometer (Olympus, Tokyo, Japan).

For TRAP, deparaffined specimens were treated with ethanolacetone (50:50, vol/vol) for $1 \mathrm{~min}$ and stained using a modified method of Akatsu et al ${ }^{9}$. Briefly, an acetate buffer ( 0.1 M sodium acetate, $\mathrm{pH}$ 5.2) containing naphthol AS-BI phosphate (Sigma) as the substrate, and fast garnet GBC salt (Sigma) as the dye for the reaction product was applied in the presence of $50 \mathrm{nM}$ sodium tartrate. Then methyl green was used for counter staining. The total number of TRAP-positive multinuclear cells subjacent to the epiphyseal plate was counted using a light microscope at $\times 200$ (about $0.5 \mathrm{~mm}^{2}$ ). The data were expressed as mean \pm standard deviation (SD) of individual rats.

4 to 7 rats were sacrificed for each group.

\section{In vitro examination}

Osteoclasts were prepared according to the method of McSheehy and Chambers ${ }^{10)}$, with modifications. Tibiae, femora and humeri were removed from 1 to 2-day-old Sprague-Dawley rats (Nihon Charles River) and dissected free of soft tissues. The bones which were removed epiphyses were minced with scissors in alpha-minimum essential medium ( $\alpha$-MEM, Dainippon Pharmaceutical Co., Japan) supplemented with 1.8 g/liter sodium bicarbonate (Wako Pure Chemical), $50 \mathrm{IU} / \mathrm{ml}$ penicillin, $50 \mu \mathrm{g} /$ $\mathrm{ml}$ streptomycin (GIBCO BRL, Grand Island, NY), $20 \mathrm{mM}$ HEPES (Wako Pure Chemical) and 10\% heat-inactivated fetal bovine serum (FBS, Bioproducts Inc., Walkviles, MD). The resulting cell suspension was centrifuged at $25 \mathrm{G}$ for $10 \mathrm{~min}$ at $4^{\circ} \mathrm{C}$, and the osteoclast-rich fraction was resuspended in the same medium. Cells were then plated on circular bovine cortical bone slices (thickness: $400 \mu \mathrm{m}$, diameter: $6 \mathrm{~mm}$ ) in each well of a 96well plate (Corning Coster Co., Cambridge, MA) or on circular coverslips (thickness:150 $\mu \mathrm{m}$, diameter:15 mm; MATSUNAMI Glass, Osaka, Japan) in 35-mm tissue culture dishes (Falcon, Oxnard, CA) at a density of $1 \times 10^{6}$ cells/bone slice or $1.5 \times 10^{6}$ cells/coverslip and preincubated at $37^{\circ} \mathrm{C}$ in a $\mathrm{CO}_{2}$ incubator $(5 \%$ $\mathrm{CO}_{2}, 95 \%$ air) for $1 \mathrm{~h}$. Then, unattached cells were rinsed away with $\alpha$-MEM and bone slices or coverslips (with attached cells) were transferred to fresh medium not containing HEPES. In some experiments, $50 \mathrm{nM}$ human parathyroid hormone (1-34) (PTH, Peptide Institute Inc., Osaka, Japan) was added with new culture medium. After $24 \mathrm{~h}$ of incubation, DMSO (Sigma) vehicle alone as control, and AZ dissolved in DMSO with a final concentration from $10^{-5}$ to $10^{-4} \mathrm{M}$ AZ was changed respectively. Then, the cells were cultured for a further 24 to $96 \mathrm{~h}$ under the above-described conditions. For each experiment, 3 to 6 bone slices or coverslips were used.

\section{Assay for osteoclastic bone resorption activity}

The cells were seeded on bone slices and incubated with AZ for $96 \mathrm{~h}$. Then, cells were removed with a brush, and resorption pits formed by osteoclasts on bone slices were stained with acid hematoxylin (Sigma) for $5 \mathrm{~min}$. Total area of resorption pits (indicator of bone resorption activity) was measured under an optical microscope.

\section{Osteoclast morphology}

Osteoclasts on bone slices or coverslips were stained for TRAP. Cells were washed with phosphate-buffered saline (PBS, $\mathrm{pH}$ 7.4), and then fixed with $10 \%$ formalin in PBS for $10 \mathrm{~min}$. After treatment with ethanol-acetone (50:50, vol/vol) for $1 \mathrm{~min}$, the surfaces of bone slices or coverslips were dried and stained for TRAP as described above. Five slide glasses were incubated for $10 \mathrm{~min}$ at room temperature. The total number of TRAP-positive multinuclear cells on each bone slice or coverslip was counted as the number of living osteoclasts.

\section{Assessment of apoptosis-like cell death}

Morphological changes and chromatin condensation were assessed by 4,6-diamidino-2-phenylindole (DAPI, CalbiochemNovabiochem Co., La Jolla, CA) staining. Cells cultured on coverslips were stained with a solution of $2 \mu \mathrm{g} / \mathrm{ml}$ DAPI in PBS for 15 minutes after staining for TRAP. Morphological changes in osteoclasts were examined under a fluorescent microscope (OPTIPHOT-2, Nikon, Tokyo, Japan). For in situ detection of DNA fragmentation, the TUNEL (TdT-mediated dUTP-biotin nick end-labeling) assay was performed on some specimens, using a TACS Blue Label ${ }^{\mathrm{TM}}$ Kit (Trevigen, Inc., Gaithersburg, MD) according to the manufacturer's instructions. Some coverslips were administered double stain with TRAP and TUNEL methods.

\section{Statistical analysis}

The values are reported as mean $\pm \mathrm{SD}$, and statistical study was performed by using Student's unpaired $t$-test.

\section{Effects of $A Z$ in vivo}

\section{Results}

Histological changes of tibiae in rats injected with AZ were examined. Width of the epiphyseal plate and cancellous tissue in the $\mathrm{AZ}$ rats were similar to those of the control rats, and no obvious 

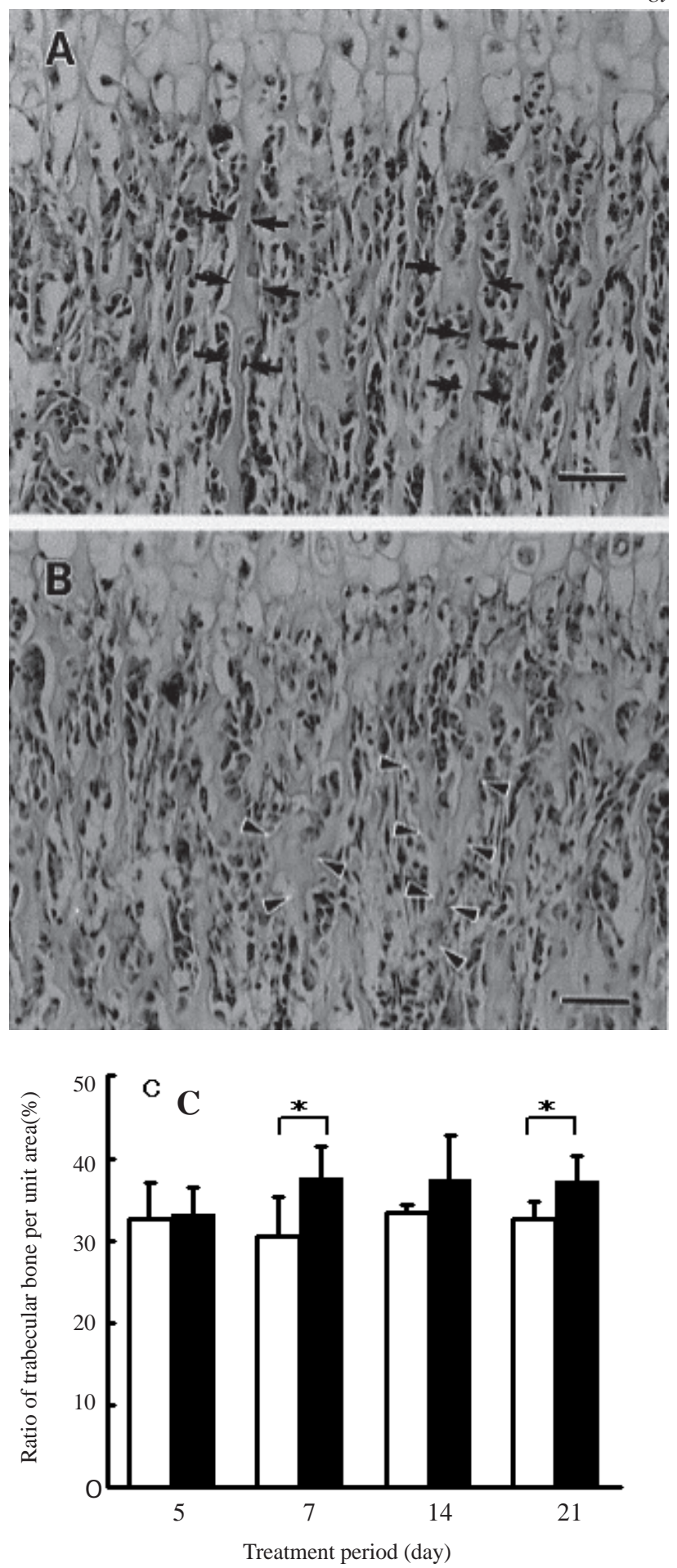

Fig. 1 Histological effects of AZ on epiphyseal plate of rat tibiae. Photomicrographs of HE staining in tibiae of rats at 21 days: control rats (A); and AZ-injected rats (B). Trabecular bone of tibiae in the control rats showed column-like structures parallel to the long axis (arrows). In the AZ-injected rats, adjacent poorly developed bone trabuculae with wide cartilage pillar were often fused together (arrow heads); bar $=50 \mu \mathrm{m}$. (C) Effects of AZ on the ratio of trabecular bone. The ratio of trabecular bone in the AZ-injected rats ( $\mathbf{\square}$ ) was significantly greater than that of the control rats $(\square)$ at 7 and 21 days after injection of AZ ( $\left.{ }^{*} \mathrm{p}<0.05\right)$.

differences in the endochondral ossification process were observed
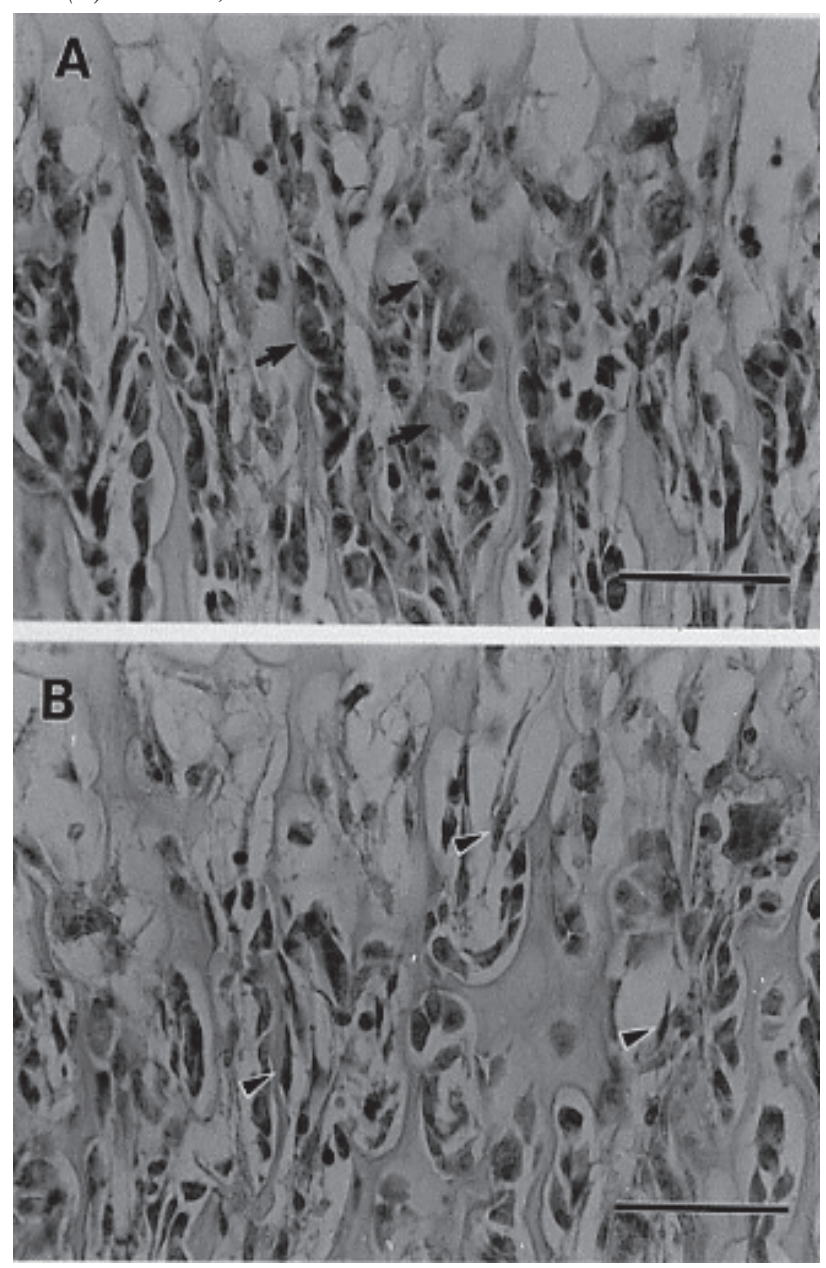

Fig. 2 Histological effects of AZ on osteoblasts.

Highly magnification photomicrographs of HE staining in tibiae of rats at cartilage-bone junction at 21 days: control rats (A); and AZ-injected rats (B). In the AZ-injected rats, osteoblats lining trabecular bone showed spindle shape (arrow heads), while those of control group showed cube shape (arrows); bar=50 $\mu \mathrm{m}$.

between these 2 groups. However, trabecular bone subjacent to the epiphyseal plate differed in structure. In the control rats, trabecular bone was laid down on the cartilage as parallel pillars of uniform width (Fig. 1A). In the AZ-treated rats, poorly developed bone trabeculae with wide cartilage pillar were fused to each other (Fig. 1B). On day 7, 14 and 21, the area of trabecular bone was greater in the AZ-treated rats than in the control rats, indicating that resorption of trabecular bone was suppressed by $\mathrm{AZ}$ (Fig. 1C). In the AZ group on 7 days after treatment, some osteoblats lining showed spindle-shaped, while those of control group showed cube-shaped (Fig. 2).

The number of osteoclasts per unit area of longitudinal bone section was also counted. In both AZ and control sections of bone, many multinucleated cells were found, which were stained for TRAP and overlaid the resorption pits. However, the number of osteoclasts per unit area was statistically lower in the AZ-treated rats than in the control rats. AZ treatment for 7, 14 and 21 days 
Chihiro Shinohara et al.: Effects of acetazolamide on osteoclasts and bone

produced about a $20 \%$ reduction in the number of osteoclasts (Fig. 3). Specifically, morphological apoptosis-like osteoclasts were not identified in bones from these animals (data not shown).

\section{Effects of $A Z$ on osteoclastic bone resorption}

The cells were placed on bone slices and cultured with PTH, followed by addition of AZ and cultured for $96 \mathrm{~h}$. In the control cultures, numerous resorption pits were formed and pit area increased with time. In the AZ cultures, pits were smaller and fewer than in the control cultures (Fig. 4A-D). A significant dosedependent decreased in pit area was detected in the presence of $10^{-5}$ to $10^{-4} \mathrm{M} \mathrm{AZ}$, and $10^{-4} \mathrm{M} \mathrm{AZ}$ inhibited bone resorption completely (Fig. 4E).

\section{Effects of AZ on the number of osteoclasts}

To determine the effects of AZ on the viability of osteoclasts, the cells were stained for TRAP. When cells were cultured with PTH on bone slices, the number of osteoclasts was reduced in a dose-dependent manner at $24 \mathrm{~h}$. At $10^{-4} \mathrm{M} \mathrm{AZ}$, the number of osteoclasts was $34 \%$ of the control (Fig. 5). At $10^{-4} \mathrm{M} \mathrm{AZ,} \mathrm{not}$ only pit formation was completely inhibited but also the number of osteoclasts was markedly reduced. Because of these results, we carried out further experiments with PTH in order to make clear the effects of AZ on new forming and existing osteoclasts as described in the materials and methods section.

A typical time course for the numbers of osteoclasts cultured on bone slices or coverslips is shown in Fig. 6. The number of osteoclasts on bone slices was decreased in AZ-treated culture compared with control cultures showing osteoclast formation despite the presence of PTH (Fig. 6A, B). The number of osteoclasts on coverslips with PTH increased with time up to 14 $\mathrm{h}$ in control culture. On the other hand, AZ-treated osteoclast was slowly decreased (Fig. 6C). Still more, the number of osteoclasts on coverslips without PTH did not increase and AZ-treated osteoclast was quickly decreased (Fig. 6D).

\section{Changes in cell morphology}

Cells cultured on coverslips were stained for TRAP and treated with the nuclear dye DAPI. There were more osteoclasts with a markedly abnormal morphology in the AZ cultures than in the control cultures. Some AZ-treated osteoclasts contained smaller, brightly stained nuclei with chromatin condensation and/or nuclear fragmentation and cytoplasmic contraction, which showed apoptosis-like cell death (Fig. 7A, B). Most of the osteoclasts in the control cultures demonstrated evidence of round and intact nuclei with diffuse homogeneous DAPI staining (Fig. 7B). TUNEL assay, a method for in situ nick end-labeling of DNA fragments in cells, was used for further examination regarding cell death of osteoclasts on coverslips. A part of osteoclast showed strong signal in the condensed nuclear fragments and downsized cytoplasm (Fig. 7C, E), while no signal and shrinking were detected on normal
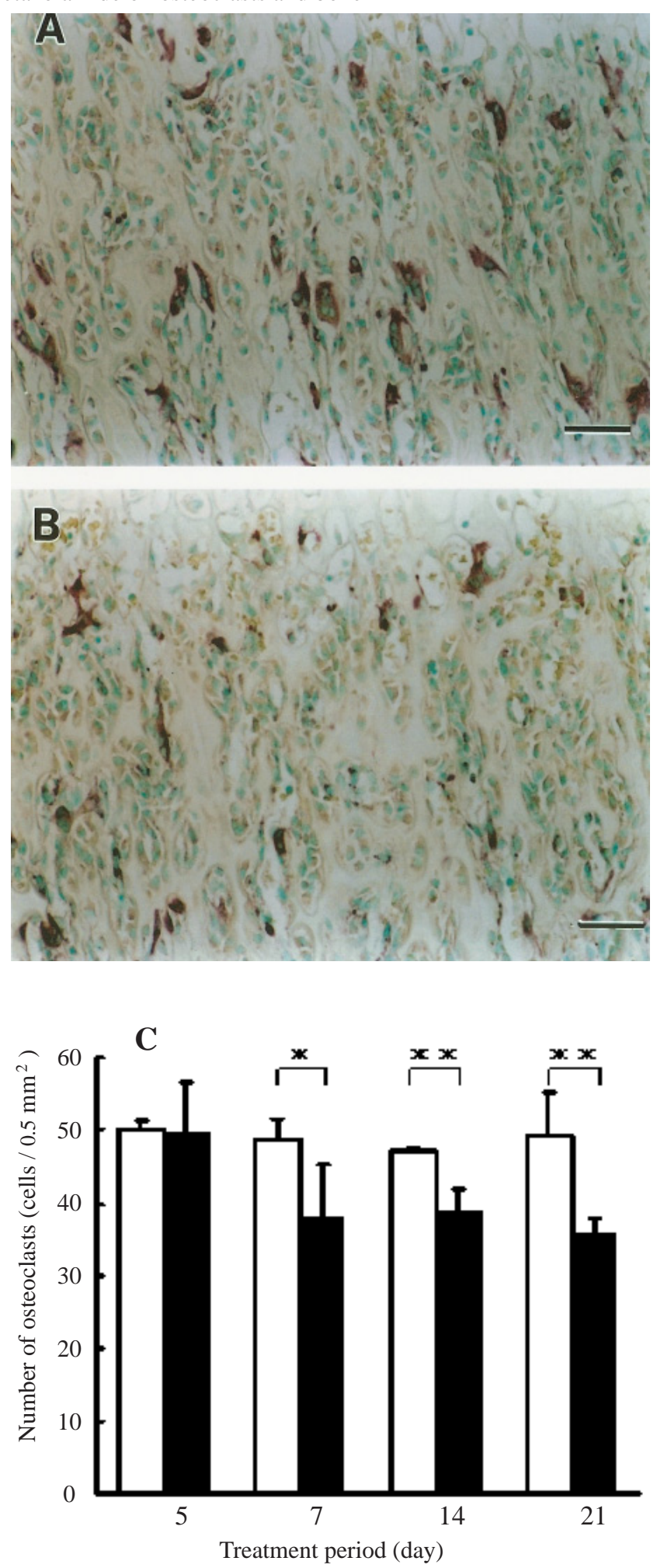

Fig. 3 Effects of AZ on the number of osteoclasts in vivo.

Photomicrographs of TRAP-stained tibiae of rats at 21 days: control rats (A); and AZ-injected rats (B); bar=50 $\mu \mathrm{m}$. The density of osteoclasts in the AZ rats ( $\square$ ) was significantly lower than that of the control rats ( $\square$ ) on day 7,14 and 21 (C) $\left({ }^{*} \mathrm{p}<0.05,{ }^{* *} \mathrm{p}<0.01\right)$.

cells (Fig. 7D, F).

The number of osteoclasts attached to coverslips demonstrated presence of nuclear fragmentation, chromatin condensation of 

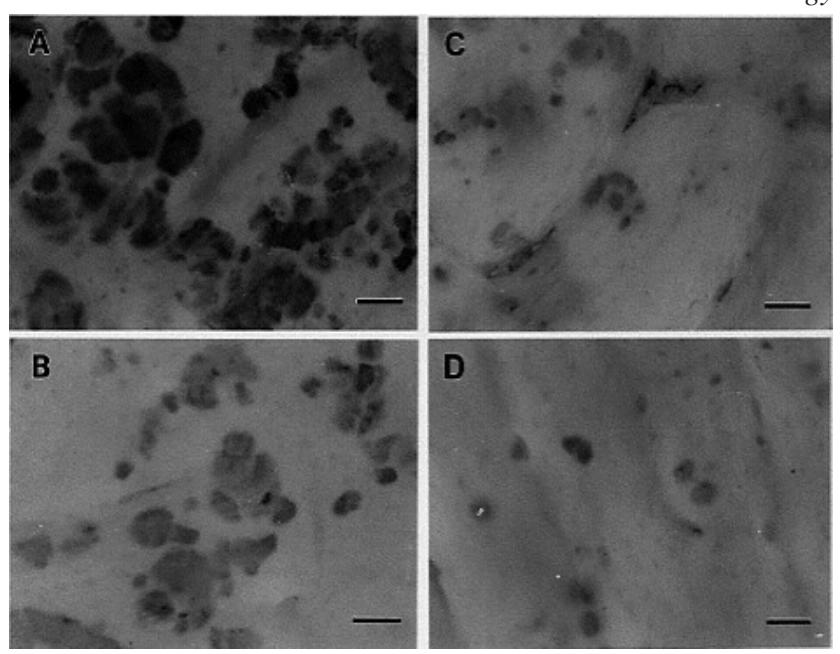

Fig. 4 Effects of AZ on resorption pits formed by osteoclasts.

Photomicrographs of resorption pits formed on bone slices are shown at 96 h of bone marrow culture (A-D). (A, control; B, 10-5 M AZ; C, 5× 10${ }^{5} \mathrm{M}$ AZ; D, $10^{-4} \mathrm{M} \mathrm{AZ}$; bar=50 $\mu \mathrm{m}$ ) The area and number of resorption pits decreased as the dose of AZ increased. Effects of AZ on the total area of resorption pits are shown (E). The total area was decreased at $96 \mathrm{~h}$ of bone marrow culture on bone slices, and bone resorption was completely inhibited by $10^{-4} \mathrm{M}$ of $\mathrm{AZ}\left({ }^{*} \mathrm{p}<0.05{ }^{* *} \mathrm{p}<0.01\right)$.

nuclei and cytoplasmic contraction showing a similar histological finding to apoptosis-like osteoclasts. At 14 or more hours of culture with and without PTH, the percentage of apoptosis-like osteoclasts had significantly increased on AZ-treated coverslips, whereas the proportion of apoptosis-like cells on control coverslips remained at the same level about $1 \%$ throughout the culture period. In the AZ-plus-PTH-treated cultures, the proportion of apoptosis-like osteoclasts was suppressed about one-third of the only AZ-treated cultures (Fig. 8).

\section{Discussion}

The effects of $\mathrm{AZ}$ on bone metabolism have been reported. $\mathrm{AZ}$ inhibit ${ }^{45} \mathrm{Ca}$ release in organ culture ${ }^{8,11)}$, suppress bone resorption ${ }^{12)}$ and increase intracellular $\mathrm{pH}$ and $\mathrm{Ca}^{2+13)}$. In the present study, AZ not only inhibited bone resorption activity by pit assay, but reduced the number of osteoclast in vivo and in vitro. Some reason why the number of osteoclasts reduced can be thought. First is promotion of cell death of osteoclasts, second is inhibition of new formation of osteoclasts and third is detachment of osteoclasts from bone surface. It is important by which caused in these decrease of osteoclasts.

In vitro study, the morphology of osteoclasts was investigated. Osteoclasts cultured with AZ clearly showed evidence of nuclear fragmentation, chromatin condensation of nuclei and cytoplasmic contraction more than that of control group. These aspects represent some histological findings of apoptosis, so it is distinct from necrosis characterized by swelling of cytoplasm and plasma membrane integrity without severe damage to nuclei ${ }^{14)}$. In the present study, it was clarified that reduction of the number of
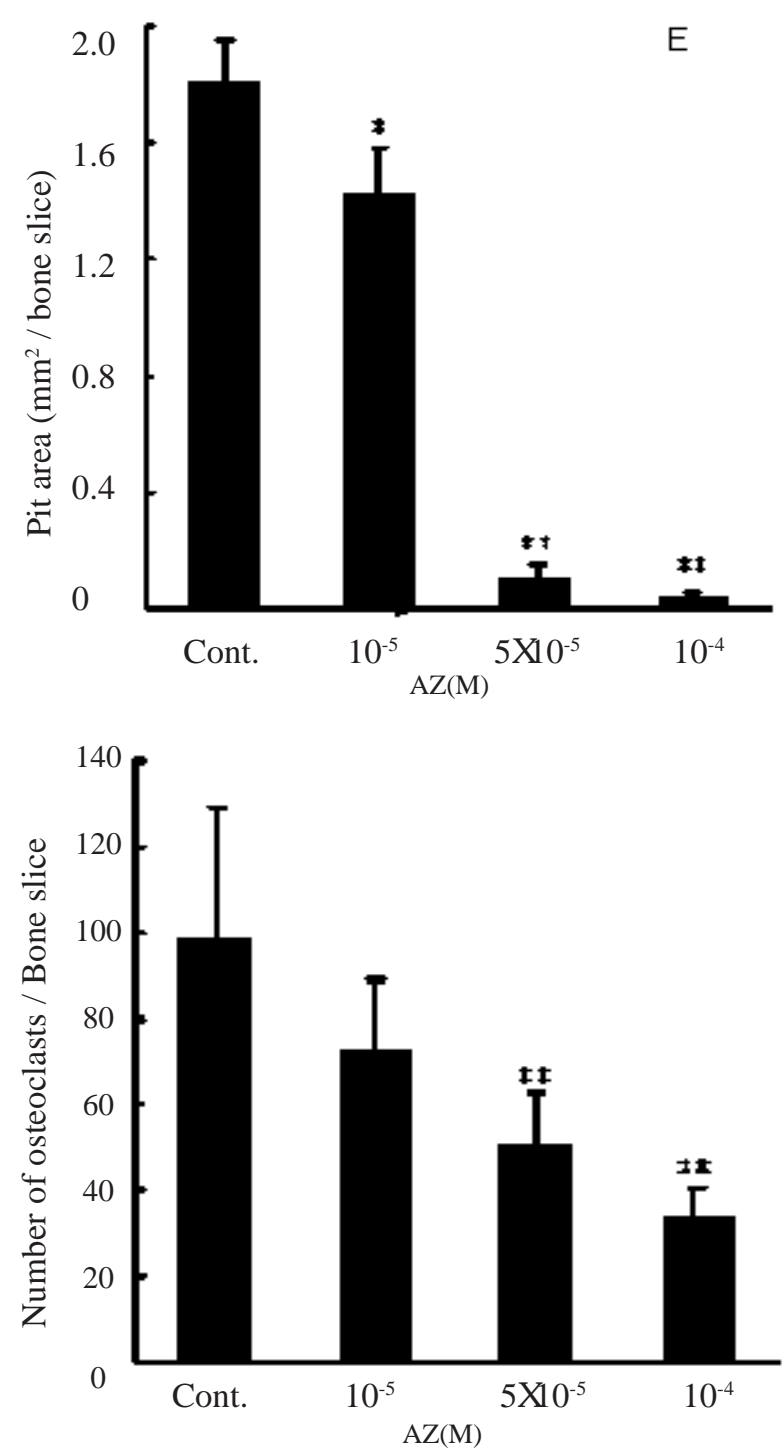

Fig. 5 Effects of AZ on the number of osteoclasts at $24 \mathrm{~h}$.

The number of osteoclasts decreased as the concentration of AZ increased $(* * \mathrm{p}<0.01)$.

osteoclasts by AZ was mainly caused by induction of cell death of osteoclasts. However, apoptosis and some necrosis share common pathway to cell death partway ${ }^{15}$, thus it is necessary to confirm this cell death carefully with the use of further evidence by annexinV stain and caspase activation. Previous studies have indicated that apoptosis is induced in osteoclasts by bisphosphonate ${ }^{16)}$, V-ATPase inhibitor ${ }^{17)}$, Vitamin $\mathrm{K}_{2}{ }^{18)}$ and estrogen ${ }^{19)}$. Though the mechanisms by which osteoclast apoptosis is induced by these substances are still unknown, however these findings suggest that inhibition of bone resorption activity of osteoclast at any step induce apoptotic cell death of osteoclast.

Osteoclasts are formed by the fusing of pre-osteoclasts, which differentiate from multipotent stem cells, and they are activated by contact with osteoblastic cells to become active bone-resorbing osteoclasts ${ }^{1-3)}$. We used bone marrow cells as the source of osteoclasts, so many kinds of cells containing osteoblastic cells 

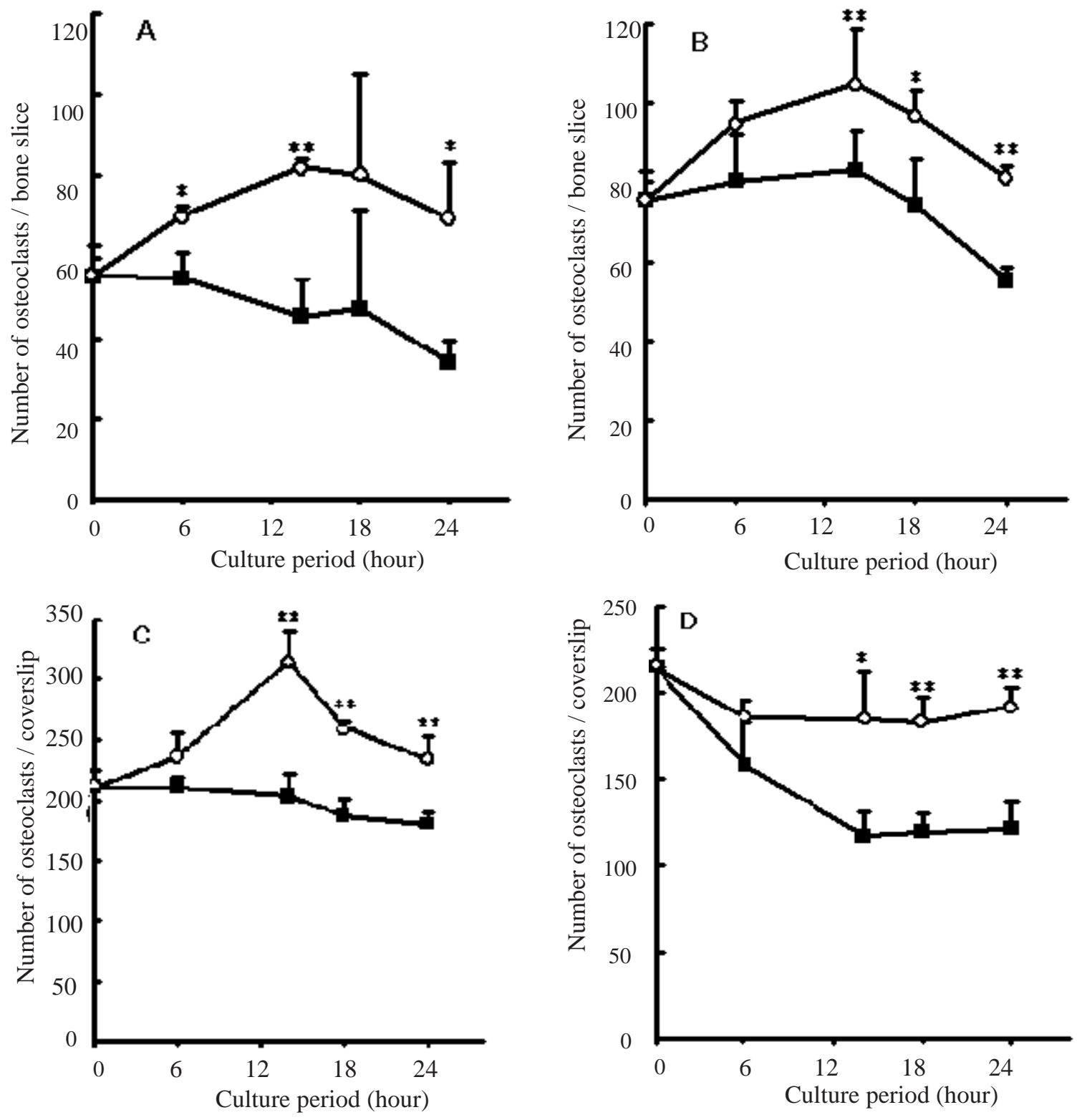

Fig. 6 Effects of AZ and PTH on the number of osteoclasts in cultures on bone slices and coverslips.

Typical time courses for the number of osteoclasts in cultures on bone slices (A, B) or coverslips (C, D) with (A, C) or without $50 \mathrm{nM}$ PTH (B, D) are shown: control cultures ( $\bigcirc$ ); and AZ-treated cultures $(\boldsymbol{\square})$. The number of osteoclasts on bone slices was decreased in AZ- treated culture, compared with control cultures despite the presence of PTH (A, B). The number of osteoclasts on coverslips with PTH increased with time up to $14 \mathrm{~h}$ in control culture. Though, AZ-treated osteoclast was slowly decreased (C). The number of osteoclasts on coverslips without PTH did not increase and AZ-treated osteoclast was quickly decreased (D). $\left({ }^{*} \mathrm{p}<0.05 * * \mathrm{p}<0.01\right)$.

existed in this culture system. These osteoblastic cells were considered to allow new osteoclast formation. Additionally PTH upregulate the expression of RANKL ${ }^{20)}$, stimulate osteoclast formation ${ }^{4)}$ and promotes bone resorption by osteoclasts ${ }^{21)}$. In the present study, the number of osteoclasts decreased even in the condition that formation of osteoclasts and inhibition of cell death were induced by PTH in bone marrow culture system, so it was indicated that reduction of the number of osteoclasts by AZ was caused by inhibiting the formation of osteoclasts too. Surely, CA
II has been detected in pre-osteoclasts ${ }^{22)}$ and AZ inhibit 1,25dihydroxyvitamin $\mathrm{D}_{3}\left[1,25(\mathrm{OH})_{2} \mathrm{D}_{3}\right]$-stimulated formation of osteoclasts ${ }^{13)}$. These findings supported our hypothesis that the decrease in osteoclast number in this study partly is due to suppressed osteoclast formation. Also PTH induces mRNA expression of CA II increased as much as 5-fold in pre-osteoclasts 23). In this study, PTH reduced the rate of apoptosis-like cell death of AZ-treated osteoclasts about one-third. Consequently, it suggested that inhibition of AZ-induced apoptosis-like cell death 

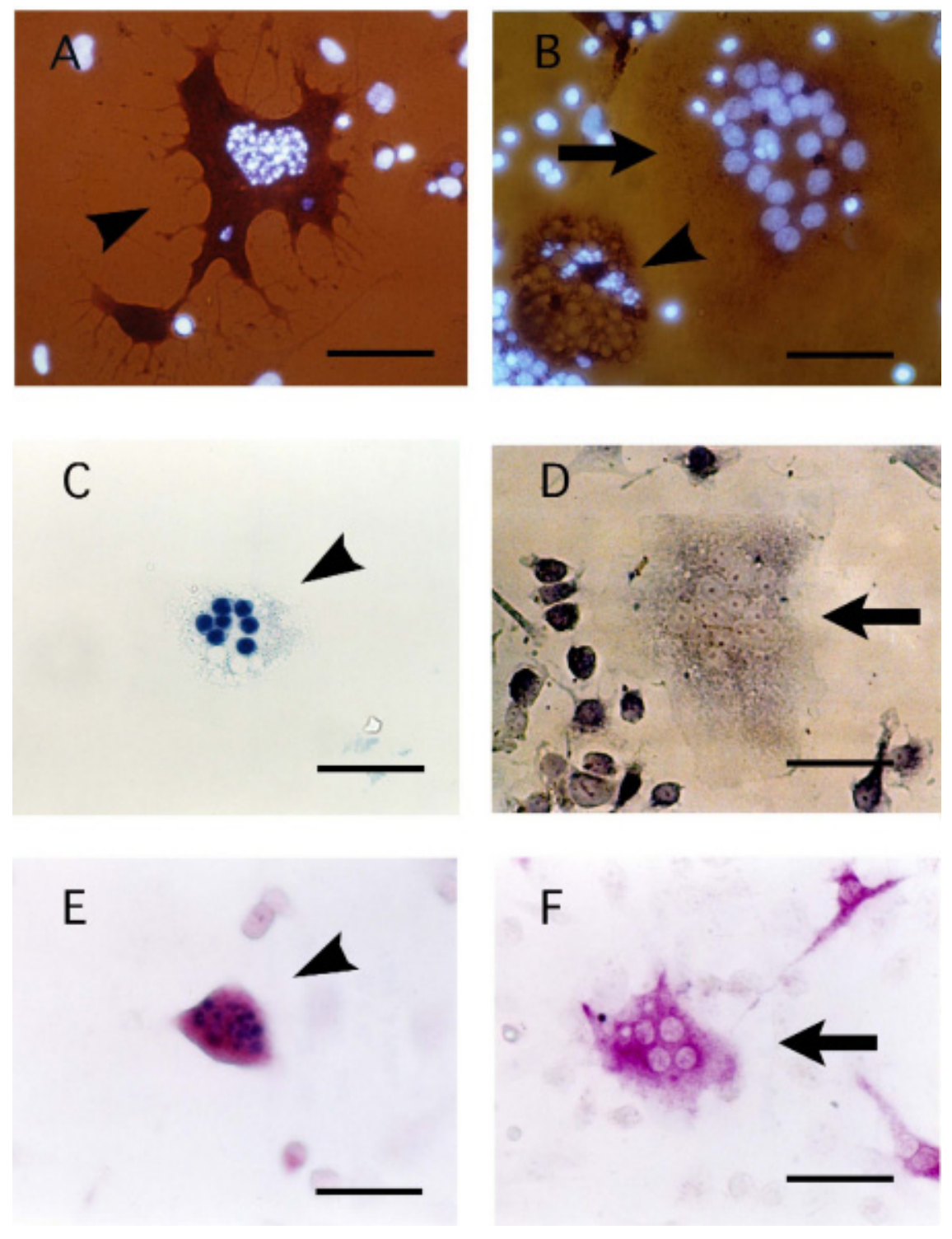

Fig. 7 Apoptosis-like change of osteoclast induced by AZ.

Osteoclasts were cultured on coverslips with $10^{-4} \mathrm{M} \mathrm{AZ}$. Fluorescence photomicrographs of apoptosis-like (A, B; arrow head) and normal (B; arrow) osteoclasts. Cells were treated with TRAP and DAPI. Apoptosis-like osteoclasts indicated bright nuclear fragments; bar=50 $\mu \mathrm{m}$. Apoptosis-like osteoclasts indicated DNA fragmentation by TUNEL assay (C; arrow head). Osteoclasts with no signal maintained normal shape (D; arrow); bar=50 $\mu \mathrm{m}$. Apoptosis-like osteoclasts stained for TRAP and TUNEL showed condensed nuclear fragments and downsized cytoplasm (E; arrow head), while no signal and shrinking were detected in normal cells (F; arrow); bar $=50 \mu \mathrm{m}$.

by PTH is a result from activated CA II and osteoclastogenesis caused by PTH.

Another possibility of decreased in the number of osteoclasts is detachment of osteoclasts from bone or coverslips. For example, it is reported that calcitonin inactivate osteoclasts by detaching from the bone surface ${ }^{24)}$. We put conditioned culture medium of each sample into the centrifuge, spun it slowly and gathered cells were assessed with a light microscope after TRAP staining. No intact or denatured osteoclasts were detected in conditioned culture medium. Podosome, a cell adhesion equipment which contain vinculin, talin and F-actin important for bone resorbing osteoclasts, exists when they cultured on both bone slices and coverslips ${ }^{25,26)}$. This result indicates that AZ decreases the number of osteoclasts not by inhibition of adhesion to the surface of culture platform materials.

From the results of previous studies applied $10^{-7}$ to $10^{-4} \mathrm{M} \mathrm{AZ}$, bone resorption was inhibited even with $10^{-7} \mathrm{M} \mathrm{AZ}^{8,13)}$. Few of cells showed any sign of necrosis in this bone marrow culture applied $10^{-5}$ to $10^{-4} \mathrm{M}$ AZ. Furthermore, dose of $10^{-4} \mathrm{M}$ AZ was not found to have a significant effect on cell proliferation and ALP activity of osteoblastic MC3T3-E1 cells derived from mouse 
Chihiro Shinohara et al.: Effects of acetazolamide on osteoclasts and bone
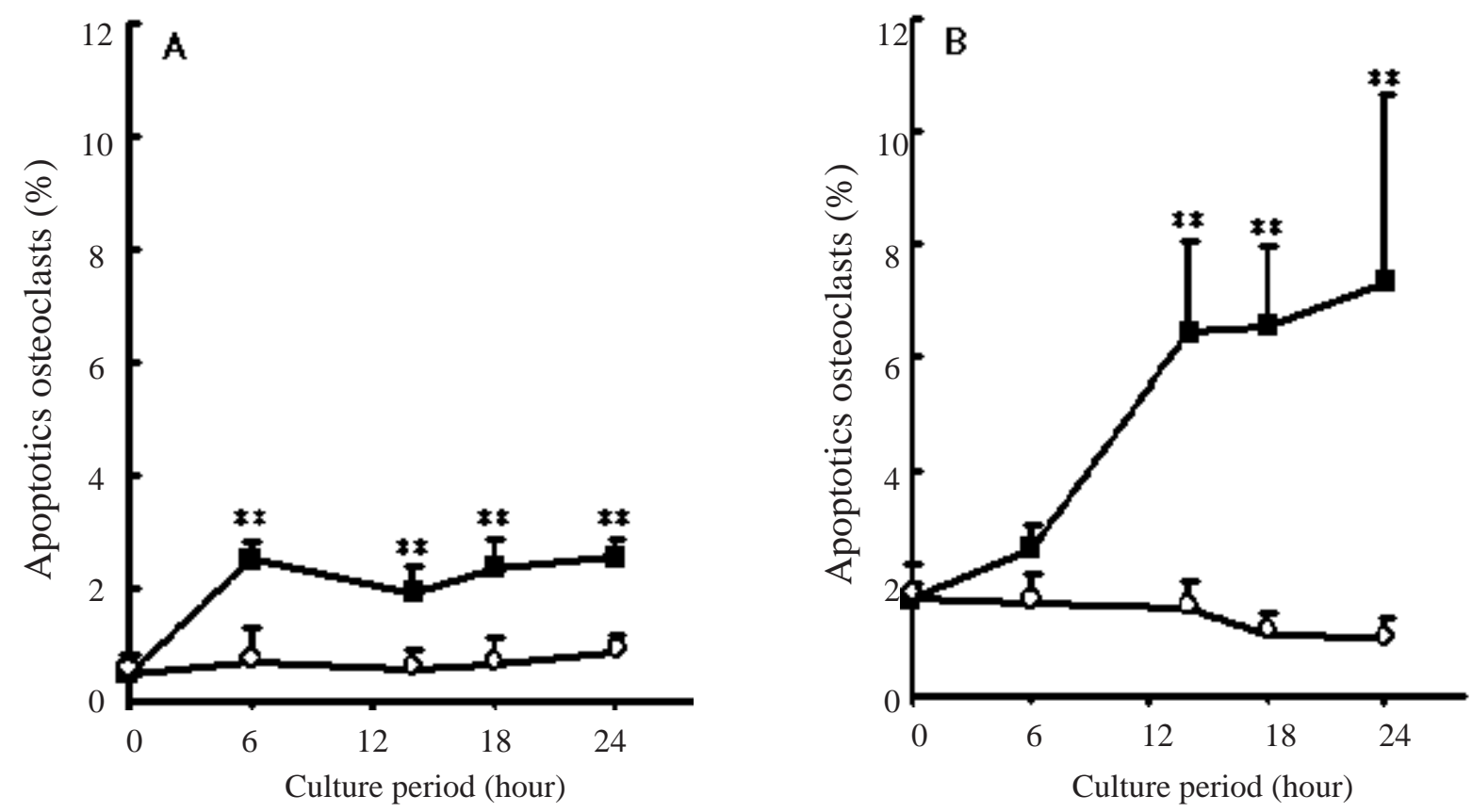

Fig. 8 Induction of apoptosis-like osteoclasts by AZ in time-dependent manner.

Cells were cultured on coverslips with (A) or without (B) $50 \mathrm{nM} \mathrm{PTH.} \mathrm{Apoptosis-like} \mathrm{osteoclasts} \mathrm{were} \mathrm{quantified} \mathrm{under} \mathrm{a} \mathrm{fluorescence}$ microscope. The rate of apoptosis-like osteoclasts in the AZ-treated cultures $(\boldsymbol{\square})$ was higher than that in the control cultures ( $\bigcirc$ ), despite the presence of PTH $\left({ }^{* *} \mathrm{p}<0.01\right)$. The rate of apoptosis-like osteoclasts in the PTH-treated cultures was lower than that of the AZtreated cultures not treated with PTH.

calvaria (data not shown). These results may provide evidence that decrease in the number of osteoclasts is not caused by cell toxicity of AZ.

In vivo experiments, $\mathrm{AZ}$ decreased the number of osteoclasts at the cartilage-bone junction and increased the ratio of trabecular bone. There is not so much of a difference between control and AZ group about width of epiphyseal plates and weight of rats through experimental period. However, it was detected that presence of spindle-shaped osteoblasts represent morphological characteristics of bone lining cells and the production of Type I collagen was reduced in AZ treated rats through immunostaining procedure (data not shown). Bone lining cells hardly engage bone formation. Type I collagen is secreted from osteoblasts and important for bone formation ${ }^{27}$ ) so appearance of osteoblasts like as bone lining cells consistent with lower Type I collagen production. Differentiation of osteoblasts is controlled by bone matrix proteins (BMPs) secreted from resorption lacunae by osteoclasts. It is considered inhibited bone resorption activity of osteoclasts lead to inactive form in osteoblasts. Increase of trabecular bone reflects not only direct result of suppressed proton secretion of osteoclasts but also inhibited of osteoclastogenesis, induced cell death and inactive osteoblasts by AZ administration. Osteoclasts with morphology that indicate apoptosis-like cell death were not detected in vivo. The reason for this may be that the duration of apoptosis was very short, which would make histological detection of apoptosis-like cell death difficult.
We indicated CA II inhibitor, AZ influence osteoclasts activity, differentiation and survival. It is reported that inhibition of VATPase-mediated proton release in osteoclasts could be a trigger for apoptosis. Then, considering the present findings about suppressed proton production, it was suggested that inhibition of proton formation and release causes apoptosis-like cell death in osteoclasts. These findings also suggest that inhibition of certain fundamental functions of cells may cause cell death. That is, it may appear that the specialized functions of certain cell types are intimately related to their survival.

\section{Acknowledgments}

We thank Dr. K. Sumitani and Dr. E. Hayashi for their technical support and invaluable advice.

\section{References}

1. Yoshida H, Hayashi S, Kunisada T, Ogawa M, Nishikawa S, Okamura H, Sudo T, Shultz LD, Nishikawa S. The murine mutation osteopetrosis is in the coding region of the macrophage colony stimulating factor gene. Nature 31;345(6274):442-444, 1990

2. Yasuda H, Shima N, Nakagawa N, Yamaguchi K, Kinosaki M, Mochizuki S, Tomoyasu A, Yano K, Goto M, Murakami A, Tsuda E, Morinaga T, Higashio K, Udagawa N, Takahashi $\mathrm{N}$, Suda T. Osteoclast differentiation factor is a ligand for osteoprotegerin/osteoclastogenesis-inhibitory factor and is identical to TRANCE/RANKL. Proc Natl Acad Sci USA 
95:3597-3602, 1998

3. Lacey DL, Timms E, Tan HL, Kelley MJ, Dunstan CR, Burgess T, Elliott R, Colombero A, Elliott G, Scully S, Hsu H, Sullivan J, Hawkins N, Davy E, Capparelli C, Eli A, Qian YX, Kaufman S, Sarosi I, Shalhoub V, Senaldi G, Guo J, Delaney J, Boyle WJ. Osteoprotegerin ligand is a cytokine that regulates osteoclast differentiation and activation. Cell 17;93(2):165176, 1998

4. Suda T, Nakamura I, Jimi E, Takahashi N. Regulation of osteoclast function. J Bone Miner Res 12(6):869-879, 1997

5. Hott, M, Marie PJ. Carbonic anhydrase activity in fetal rat bone resorbing cells: inhibition by acetazolamide infusion. $\mathrm{J}$ Develop Physiol 12:277-281, 1989

6. Nakamura H, Moriyama Y, Futai M, Ozawa H. Immunohistochemical localization of vacuolar $\mathrm{H}(+)$-ATPase in osteoclasts of rat tibiae. Arch Histol Cytol 57(5):535-539, 1994

7. McMahon C, Will A, Hu P, Shah GN, Sly WS, Smith OP. Bone marrow transplantation corrects osteopetrosis in the carbonic anhydrase II deficiency syndrome. Blood 97(7):1947-1950, 2001

8. Raisz LG, Simmons HA, Thompson WJ, Shepard KL, Anderson PS, Rodan GA. Effects of a potent carbonic anhydrase inhibitor on bone resorption in organ culture. Endocrinology 122(3):1083-1086, 1988

9. Akatsu T, Tamura T, Takahashi N, Udagawa N, Tanaka S, Sasaki T, Yamaguchi A, Nagata N, Suda T. Preparation and characterization of a mouse osteoclast-like multinucleated cell population. J Bone Miner Res 7(11):1297-1306, 1992

10. McSheehy PMJ, Chambers TJ. 1,25-dihydroxyvitamin $\mathrm{D}_{3}$ stimulates rat osteoblastic cells to release a soluble factor that increases osteoclastic bone resorption. J Clin Invest 80:425429, 1987

11. Minkin C, Jennings JM. Carbonic anhydrase and bone remodeling: sulfonamide inhibition of bone resorption in organ culture. Science 176:1031-1033, 1972

12. Hall TJ, Higgins W, Tardif C, Chambers TJ. A comparison of the effects of inhibitors of carbonic anhydrase on osteoclastic bone resorption and purified carbonic anhydrase isozyme II. Calcif Tissue Int 49:328-332, 1991

13. Lehenkari P, Hentunen TA, Laitala-Leinonen T, Tuukkanen J, Vaananen HK. Carbonic anhydrase II plays a major role in osteoclast differentiation and bone resorption by effecting the steady state intracellular $\mathrm{pH}$ and $\mathrm{Ca}^{2+}$. Exp Cell Res 242:128137, 1998

14. Stewart BW. Mechanisms of apoptosis: integration of genetic, biochemical, and cellular indicators. J Natl Cancer Inst 86(17):1286-1296, 1994

15. Eguchi Y, Shimizu S, Tsujimoto Y. Intracellular ATP levels determine cell death fate by apoptosis or necrosis. Cancer Res 57(10):1835-1840, 1997

16. Hughes DE, Wright KR, Uy HL, Sasaki A, Yoneda T, Roodman
GD, Mundy GR, Boyce BF. Bisphosphonates promote apoptosis in murine osteoclasts in vitro and in vivo. $\mathrm{J}$ Bone Miner Res 10(10):1478-1487, 1995

17. Okahashi N, Nakamura I, Jimi E, Koide M, Suda T, Nishihara T. Specific inhibitors of vacuolar $\mathrm{H}^{+}$-ATPase trigger apoptotic cell death of osteoclasts. J Bone Miner Res 12(7):1116-1123, 1997.

18. Kameda T, Miyazawa K, Mori Y, Yuasa T, Shiokawa M, Nakamaru Y, Mano H, Hakeda Y, Kameda A, Kumegawa M. Vitamin $\mathrm{K}_{2}$ inhibits osteoclastic bone resorption by inducing osteoclast apoptosis. Biochem Biophys Res Commun 220:515-519, 1996

19. Kameda T, Mano H, Yuasa T, Mori Y, Miyazawa K, Shiokawa M, Nakamaru Y, Hiroi E, Hiura K, Kameda A, Yang NN, Hakeda Y, Kumegawa M. Estrogen inhibits bone resorption by directly inducing apoptosis of the bone-resorbing osteoclasts. J Exp Med 186(4):489-495, 1997

20. Fu Q, Jilka RL, Manolagas SC, O’Brien CA. Parathyroid hormone stimulates receptor activator of NFkappa B ligand and inhibits osteoprotegerin expression via protein kinase A activation of cAMP-response element-binding protein. J Biol Chem 277(50):48868-48875, 2002

21. Takada Y, Kusuda M, Hiura K, Sato T, Mochizuki H, Nagao Y, Tomura M, Yahiro M, Hakeda Y, Kawashima H, Kumegawa M. A simple method to assess osteoclast-mediated bone resorption using unfractionated bone cells. Bone Miner 17(3):347-359, 1992

22. Huang WH, Lau AT, Daniels LL, Fujii H, Seydel U, Wood DJ, Papadimitriou JM, Zheng MH. Detection of estrogen receptor a, carbonic anhydrase II and tartrate-resistant acid phosphatase mRNAs in putative mononuclear osteoclast precursor cells of neonatal rats by fluorescence in situ hybridization. J Mol Endocrinol 20(2):211-219, 1998

23. Biskobing DM, Fan D, Fan X, Rubin J. Induction of carbonic anhydrase II expression in osteoclast progenitors requires physical contact with stromal cells. Endocrinology 138(11):4852-4857, 1997

24. Akisaka T, Gay CV. An ultracytochemical investigation of ouabain-sensitive p-nitrophenylphosphatase in chick osteoclasts. Cell Tissue Res 244(1):57-62, 1986

25. Kanehisa J, Yamanaka T, Doi S, Turksen K, Heersche JN, Aubin JE, Takeuchi H. A band of F-actin containing podosomes is involved in bone resorption by osteoclasts. Bone 11(4):287-293, 1990

26. Zambonin-zallone A, Teti A, Carano A, Marchisio PC. The distribution of podosomes in osteoclasts cultured on bone laminae: effect of retinol. J Bone Miner Res 3(5):517-523, 1988

27. Rodan GA. Introduction to bone biology. Bone 13 Suppl 1:S36, 1992 